\title{
The effects analysis of two neonicotinoid insecticides on in vitro maturation of porcine oocytes using hanging drop monoculture method
}

\author{
Sadamasa ISHIKAWA ${ }^{1)}$, Kou HIRAGA ${ }^{1)}$, Yuuki HIRADATE ${ }^{1)}$ and Kentaro TANEMURA ${ }^{1) *}$ \\ ${ }^{1)}$ Laboratory of Animal Reproduction and Development, Graduate School of Agricultural Science, Tohoku University, Sendai 981-8555, \\ Japan
}

(Received 5 January 2015/Accepted 24 January 2015/Published online in J-STAGE 6 February 2015)

ABSTRACT. Acetamiprid (ACE) and imidacroprid (IMI) are known neonicotinoid insecticides with strong affinities for the insect-selective nicotinic acetylcholine receptor. These provide insect control by hyperstimulating insect nerves and are used for agricultural pest management. However, it has also been reported that ACE and IMI affect mammalian reproductive function. We determined the effects of ACE and IMI on the in vitro maturation of porcine oocytes. Significant decreases in nuclear maturation rates were observed in the ACE or IMIexposed groups. Also, in matured oocytes from the ACE or IMI-exposed groups, irregular chromosomes were observed. Our results suggest that ACE and IMI exposure was detrimental to porcine oocytes and the extent of the effects depends on the concentration of exposure. KEY WORDS: hanging drop, in vitro maturation (IVM), neonicotinoid, porcine oocyte

doi: 10.1292/jvms.15-0008; J. Vet. Med. Sci. 77(6): 725-728, 2015

Neonicotinoid insecticides show strong affinities for the insect-selective nicotinic acetylcholine receptor (nAChR) and control insects by hyperexcitation of their nerves, and their effective insect control has been used to protect many kinds of crops and animal health $[10,17]$.

Recently, however, two of the main compounds in neonicotinoid, acetamiprid (ACE) and imidacloprid (IMI), were reported to have comparable effects at the mammalian $\mathrm{nAChR}$, and their safety was called into question [8]. The negative effects of exposure to these compounds are unclear, particularly prenatal exposure.

In the past, there have been some reports of the influence of germ line on nicotine exposure. Specifically, the influence of ACE and IMI in mouse oocytes and human sperm has been reported $[2,20]$.

Furthermore, it was reported that ACE and IMI affect reproductive function. Kapoor et al. (2011) reported changes in ovary morphology following IMI exposure [7].

However, there are few reports about the direct effects on mammalian germ line by ACE and IMI. In later years, Gu et $a l$. and Rasgele PG reported the effects of ACE and IMI in mouse sperm, but there are no reports in oocyte that are from a female germ cell $[3,14]$.

Although there is concern that domestic animals, such as porcine, are exposed to these neonicotinoid insecticides, there are limited data available.

We developed a screening system for high throughput

*Correspondence to: Tanemura, K., Laboratory of Animal Reproduction and Development, Graduate School of Agricultural Science, Tohoku University, Sendai 981-8555, Japan.

e-mail: kentaro@m.tohoku.ac.jp

(C)2015 The Japanese Society of Veterinary Science

This is an open-access article distributed under the terms of the Creative Commons Attribution Non-Commercial No Derivatives (by-nc-nd) License $<$ http://creativecommons.org/licenses/by-nc-nd/3.0/>. characteristics using the Hanging Drop (HD) monoculture system for analyzing of the effects in one oocyte. The HD method that we used in this study is a culture method that makes use of a small drop in a dish base and inversion of the dish to be used for culture of the embryoid body [9] and ovarian follicle [1]. This method is new and contributed to the screening of effective material for culturing porcine oocytes to in vitro maturation (IVM) [6].

In this study, using a screening system, we analyzed the effects of ACE and IMI addition to IVM medium on nuclear maturation in porcine oocytes.

Ovaries were collected from prepubertal gilts at a local slaughterhouse, transported to the laboratory in a container within $2 \mathrm{hr}$ of removal and placed in saline warmed to $37^{\circ} \mathrm{C}$. The follicular fluid and porcine oocytes were aspirated from antral follicles (diameter: 3-6 mm) with a $10 \mathrm{ml}$ syringe attached to an 18 gauge needle. Compact cumulus-oocyte complexes (COCs) with uniform ooplasm were selected in phosphate-buffered saline (PBS; Nissui Pharmaceutical, Tokyo, Japan) and supplemented with $0.1 \%$ polyvinyl alcohol (PVA; Sigma Chemical, St. Louis, MO, U.S.A.). The culture method referred to the report of Hiraga et al. (2013) [5]. After washing three times in $0.1 \%$ PBS-PVA, the COCs were cultured in $10 \mu l(10 \mu l$ of medium/1 COC) of NCSU-23 medium [12] supplemented with $50 \mu \mathrm{M} \beta$-mercaptoethanol (Sigma), $0.6 \mathrm{mM}$ cysteine (Sigma), 0.5\% insulin (GibcoBRL, Cergy Pontoise, France; Cat. 18125), 10\% (v/v) porcine follicular fluid, 10 IU pregnant mare serum gonadotropin (PMSG; Serotropin; Teikokuzouki, Tokyo, Japan), 10 IU human chorionic gonadotropin (hCG; Puberogen; Sankyo, Tokyo, Japan) and $1 \mathrm{mM}$ dibutyryl cyclic AMP (Sigma) for the first $22 \mathrm{hr}$ of maturation at $38.5^{\circ} \mathrm{C}$ in $5 \% \mathrm{CO}_{2}$ in air. Then, the COCs were cultured for $22 \mathrm{hr}$ in the medium without hormones or dbcAMP. Each droplet of medium was overlaid with liquid paraffin (NACALAI TESQUE, INC., Kyoto, Japan). 
After $44 \mathrm{hr}$ of incubation, denuded oocytes (DOs) were prepared from some of the incubated COCs by removing cumulus cells through gentle vortexing in PB1 medium [13] that contained $0.1 \%$ hyaluronidase (Sigma). DOs were mounted on glass slides and then fixed in acetic acid: ethanol (1:3) for $48 \mathrm{hr}$. The fixed oocytes were stained with $1 \%$ aceto-orcein and observed under a phase-contrast microscope to evaluate nuclear status

We used the HD method for the IVM experiments. In the HD method, $10 \mu \mathrm{l}$ of the culture medium were placed in each well of a 96-well plate (Thermo Fisher Scientific Inc., Waltham, MA, U.S.A.), and each droplet of medium was overlaid with liquid paraffin. After $1 \mathrm{COC}$ was placed in the medium, the plate was inverted.

COCs were cultured with various concentrations of ACE (Wako Pure Chemical Industries, Ltd., Osaka, Japan) or IMI (Wako) prepared using dimethyl sulfoxide (NACALAI TESQUE) $(\leq 0.1 \%)$, at finally determined concentrations (10, 30 and $100 \mathrm{ppm})$ for $44 \mathrm{hr}$. After $44 \mathrm{hr}$ of total incubation, all oocytes were fixed. Nuclear status was analyzed, and the percentages of oocyte at MII stage relative to all of the cultured oocytes were calculated.

The range of concentrations of tested chemicals was set on a pesticide residue reference value in Japan, and the minimum of pesticide residue reference value is that ACE is 30 ppm and IMI is $10 \mathrm{ppm}$. Also, in this study, $100 \mathrm{ppm}$ was set as the maximum.

Each experiment was replicated at least three times. Data are presented as means \pm standard error (SE). Statistical analyses were carried out using analysis of variance (ANOVA) and Fisher's protected least significant difference test. A $P$ value $<0.01$ was considered to indicate significant differences $(* * P<0.01, * P<0.05)$.

The results showed that the nuclear maturation rate in the ACE-exposed group was 10 ppm $(73.58 \pm 4.84 \%)$, which was not significantly different from that of the control group $(78.46 \pm 3.85 \%)$. However, in the ACE-exposed groups at $30 \mathrm{ppm}$ and $100 \mathrm{ppm}(66.76 \pm 4.12 \%, 53.85 \pm 2.32 \%)$, a significant decrease in the nuclear maturation rate was observed compared with the control group. Conversely, when the IMI was added at all doses, the nuclear maturation rate was significantly lower than the control (10 ppm: $66.09 \pm 5.81 \%$, 30 ppm: $61.17 \pm 8.90 \%$ and 100 ppm: $56.52 \pm 7.53 \%)$. Furthermore, when the ACE-exposed group was compared with the IMI-exposed group at $100 \mathrm{ppm}$, the nuclear maturation of the ACE-exposed group tended to be lower than the IMIexposed group $(P \approx 0.55)$ (Fig. 1$)$.

In normal metaphase II oocytes (control group), localized chromosomes of oocyte and polar body were observed as shown in Fig. 2A. But, in metaphase II oocytes which exposed with ACE or IMI groups, the chromosomes were dispersed (irregular chromosomes) (Fig. 2B and 2C). These oocytes were observed in ACE-exposed groups at 30-100 ppm and IMI-exposed groups at 10-100 ppm.

Although it is generally thought that neonicotinoid insecticides have low affinity for mammalian nAChRs, in ACE and IMI, two of the main compounds in neonicotinoid, it has been reported that ACE and IMI affect mammalian nAChR

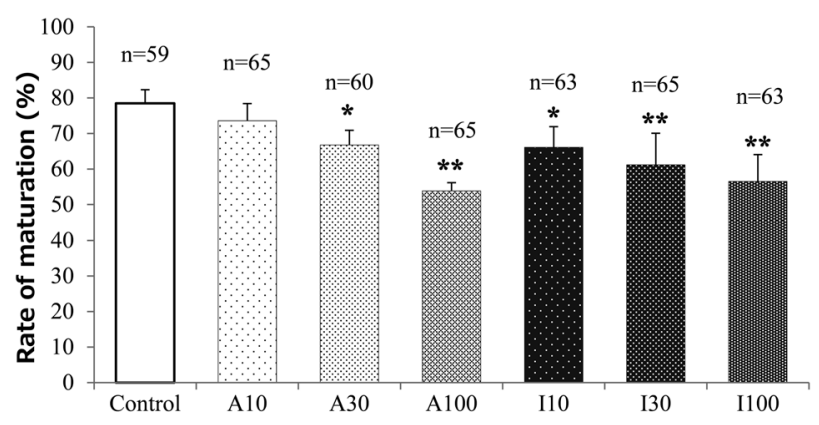

Fig. 1. Effects on nuclear maturation by ACE and IMI (10, 30, 100 $\mathrm{ppm}$ ) addition to the in vitro maturation medium. Data are presented as means \pm standard deviations. Different letters indicate significant differences among the treatments. ${ }^{*} P<0.05$, $* * P<0.01$.

at the same level as nicotine [8]. In addition, it has also been reported that these have effects on reproduction [3, 14]. Thus, in this study, we evaluated porcine oocytes that were exposed to neonicotinoid insecticides. Finally, we analyzed effects on porcine oocyte IVM from addition of ACE and IMI to IVM medium during nuclear maturation.

Our results show that the nuclear maturation rate of the ACE-exposed group at $10 \mathrm{ppm}$ was not significantly different, although a significant decrease in nuclear maturation rate was observed in the IMI-exposed group at $10 \mathrm{ppm}$. From the above, we suggest that the threshold of effects of IMI is lower than that of ACE in porcine oocyte IVM. Also, although a significant difference was observed in ACE or IMI-exposed groups at $30 \mathrm{ppm}$, this difference in IMI-exposed group was bigger than in the ACE-exposed group. Conversely, a significant difference $(P<0.01)$ was observed in the ACE or IMI-exposed groups at $100 \mathrm{ppm}$, and the nuclear maturation of the ACE-exposed group became lower than the IMIexposed group. Therefore, we suggest that nuclear maturation is inhibited in ACE at more than $30 \mathrm{ppm}$, and in IMI at more than $10 \mathrm{ppm}$. It is likely that ACE has more detrimental effects than IMI above $100 \mathrm{ppm}$. Additionally, an irregular chromosome was observed in metaphase II oocytes for ACE and IMI-exposed groups. The IMI-exposed group showed these effects at a lower concentration $(10 \mathrm{ppm})$ than the ACE exposed group (30 ppm). We suggest that IMI is more detrimental than $\mathrm{ACE}$, at low concentrations in porcine oocyte IVM.

The mechanism of the effects of ACE and IMI on porcine oocyte maturation is not clear. However, in rat ovaries from animals exposed to IMI by oral administration, a significant increase of lipid peroxide (LPO) is caused by decreases of the antioxidant glutathione and antioxidative enzyme activity including superoxide dismutase, catalase and glutathione peroxidase [7]. The huge amount of lipid in a porcine oocyte, compared with oocytes from other mammalian species, is mostly made up of triglycerides (TGs) [11, 15, 16]. TG plays an important role in energy metabolism by providing adenosine triphosphate (ATP) for protein synthesis, meiosis and cytoplasmic maturation [15]. In addition, we recognize that TG is oxidized to LPO [4]. LPO generally damages 

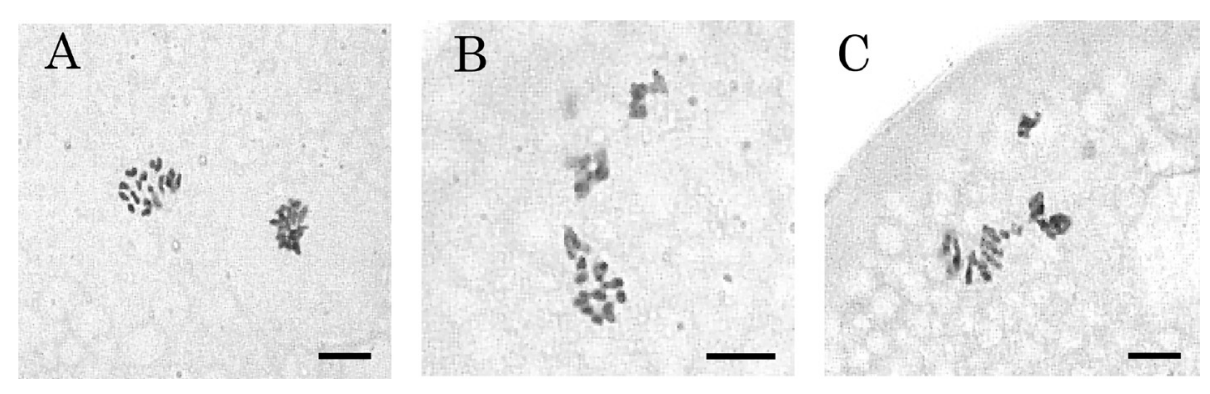

Fig. 2. Chromosomes in metaphase II oocytes. (A) control, (B) acetamiprid exposed groups, (C) imidacloprid exposed groups (Orcein staining). Bar $=5 \mu \mathrm{m}$

DNA and is known as a cause of cancer [19]. Also, Yazaki et al. reported that the nuclear maturation rate becomes lower as the ratio of LPO in porcine oocyte increases [18]. We reported that a significant increase of nuclear maturation was caused by addition of antioxidants to IVM medium in porcine oocyte IVM [6], suggesting the damage of oxidative stress on porcine oocyte IVM is serious.

Therefore, the decrease of nuclear maturation was likely caused by the influence of oxidative stress, from TG becoming oxidized to LPO in oocytes by direct exposure to IMI. However, in ACE and IMI, it is thought that a more detailed influence analysis using porcine oocyte is necessary.

The HD monoculture system used for this study had high-throughput efficiency, and we succeed in rapid screening of effective material in porcine oocytes on IVM [6]. We screened ACE and IMI as these are believed to have adverse effects that can be detected using the HD monoculture system. As a result, we could detect reproductive toxicity that inhibited nuclear maturation in porcine oocyte. Therefore, the screening system was useful for assessing the effects of exposure to chemical substances in porcine oocyte IVM. We suggest this screening system could contribute to livestock production and improve the productivity of domestic animal agriculture by functioning as an index of safety assessment for agricultural chemicals.

ACKNOWLEDGMENTS. We thank the staff of Meat Inspection Office, Sendai, Japan, for supplying the porcine ovaries.

\section{REFERENCES}

1. Choi, J. K., Agarwal, P. and He, X. 2013. In vitro culture of early secondary preantral follicles in hanging drop of ovarian cellconditioned medium to obtain MII oocytes from outbred deer mice. Tissue Eng. Part A 19: 2626-2637. [Medline] [CrossRef]

2. Gandini, L., Lombardo, F., Lenzi, A., Culasso, F., Pacifici, R., Zuccaro, P. and Dondero, F. 1997. The in-vitro effects of nicotine and cotinine on sperm motility. Hum. Reprod. 12: 727-733. [Medline] [CrossRef]

3. Gu, Y. H., Li, Y., Huang, X. F., Zheng, J. F., Yang, J., Diao, H., Yuan, Y., Xu, Y., Liu, M., Shi, H. J. and Xu, W. P. 2013. Reproductive effects of two neonicotinoid insecticides on mouse sperm function and early embryonic development in vitro. PLoS ONE 8: e70112. [Medline] [CrossRef]

4. Gutteridge, J. M. 1994. Biological origin of free radicals, and mechanisms of antioxidant protection. Chem. Biol. Interact. 91: 133-140. [Medline] [CrossRef]

5. Hiraga, K., Hoshino, Y., Tanemura, K. and Sato, E. 2013. Selection of in vitro-matured porcine oocytes based on localization patterns of lipid droplets to evaluate developmental competence. J. Reprod. Dev. 59: 405-408. [Medline] [CrossRef]

6. Ishikawa, S., Machida, R., Hiraga, K., Hiradate, Y., Suda, Y. and Tanemura, K. 2014. Hanging drop monoculture for selection of optimal antioxidants during in vitro maturation of porcine oocytes. Reprod. Domest. Anim. 49: e26-e30. [Medline] [CrossRef]

7. Kapoor, U., Srivastava, M. K. and Srivastava, L. P. 2011. Toxicological impact of technical imidacloprid on ovarian morphology, hormones and antioxidant enzymes in female rats. Food Chem. Toxicol. 49: 3086-3089. [Medline] [CrossRef]

8. Kimura-Kuroda, J., Komuta, Y., Kuroda, Y., Hayashi, M. and Kawano, H. 2012. Nicotine-like effects of the neonicotinoid insecticides acetamiprid and imidacloprid on cerebellar neurons from neonatal rats. PLoS ONE 7: e32432. [Medline] [CrossRef]

9. Lee, W. G., Ortmann, D., Hancock, M. J., Bae, H. and Khademhosseini, A. 2010. A hollow sphere soft lithography approach for long-term hanging drop methods. Tissue Eng. Part C Methods 16: 249-259. [Medline] [CrossRef]

10. Matsuda, K., Buckingham, S. D., Kleier, D., Rauh, J. J., Grauso, M. and Sattelle, D. B. 2001. Neonicotinoids: insecticides acting on insect nicotinic acetylcholine receptors. Trends Pharmacol. Sci. 22: 573-580. [Medline] [CrossRef]

11. McEvoy, T. G., Coull, G. D., Broadbent, P. J., Hutchinson, J. S. and Speake, B. K. 2000. Fatty acid composition of lipids in immature cattle, pig and sheep oocytes with intact zona pellucida. J. Reprod. Fertil. 118: 163-170. [Medline] [CrossRef]

12. Petters, R. M. and Wells, K. D. 1993. Culture of pig embryos. $J$. Reprod. Fertil. Suppl. 48: 61-73. [Medline]

13. Quinn, P., Barros, C. and Whittingham, D. G. 1982. Preservation of hamster oocytes to assay the fertilizing capacity of human spermatozoa. J. Reprod. Fertil. 66: 161-168. [Medline] [CrossRef]

14. Rasgele, P. G. 2014. Abnormal sperm morphology in mouse germ cells after short-term exposures to acetamiprid, propineb, and their mixture. Arh. Hig. Rada Toksikol. 65: 47-56. [Medline] [CrossRef]

15. Sturmey, R. G. and Leese, H. J. 2003. Energy metabolism in pig oocytes and early embryos. Reproduction 126: 197-204. [Medline] [CrossRef]

16. Sturmey, R. G., Reis, A., Leese, H. J. and McEvoy, T. G. 2009. Role of fatty acids in energy provision during oocyte maturation and early embryo development. Reprod. Domest. Anim. 44 Suppl 3: 50-58. [Medline] [CrossRef]

17. Tomizawa, M. and Casida, J. E. 2005. Neonicotinoid insecticide 
toxicology: mechanisms of selective action. Annu. Rev. Pharmacol. Toxicol. 45: 247-268. [Medline] [CrossRef]

18. Yazaki, T., Hiradate, Y., Hoshino, Y., Tanemura, K. and Sato, E. 2013. L-carnitine improves hydrogen peroxide-induced impairment of nuclear maturation in porcine oocytes. Anim. Sci. J. 84: 395-402. [Medline] [CrossRef]

19. Winczura, A., Zdżalik, D. and Tudek, B. 2012. Damage of DNA and proteins by major lipid peroxidation products in genome stability. Free Radic. Res. 46: 442-459. [Medline] [CrossRef]

20. Zenzes, M. T. and Bielecki, R. 2004. Nicotine-induced Disturbances of Meiotic Maturation in Cultured Mouse Oocytes: Alterations of Spindle Integrity and Chromosome Alignment. Tob. Induc. Dis. 2: 151-161. [Medline] [CrossRef] 